\title{
Extraction of amphoteric amino acid by bipolar membrane electrodialysis: Methionine acid as a case study
}

\section{Supplementary information}

Xi Lin ${ }^{1}$, Jiefeng Pan ${ }^{1}$, Mali Zhou ${ }^{1}$, Yanqing Xu ${ }^{1}$, Jiuyang Lin $^{2} *$,Jiangnan Shen ${ }^{1}$, Congjie Gao ${ }^{1}$, Bart Van der Bruggen ${ }^{3}$

1. Center for Membrane Separation and Water Science \& Technology, Zhejiang University of Technology, Hangzhou, 310014, China

2. School of Environment and Resources, Qi Shan Campus, Fuzhou University, No.2 Xueyuan Road, University Town, 350108 Fuzhou, Fujian, China

3. Department of Chemical Engineering, KU Leuven, W. de Croylaan 46, B-3001 Leuven, Belgium

* Corresponding authors: linjiuyang@126.com (J. Lin); shenjn@zjut.edu.cn (J. Shen) 


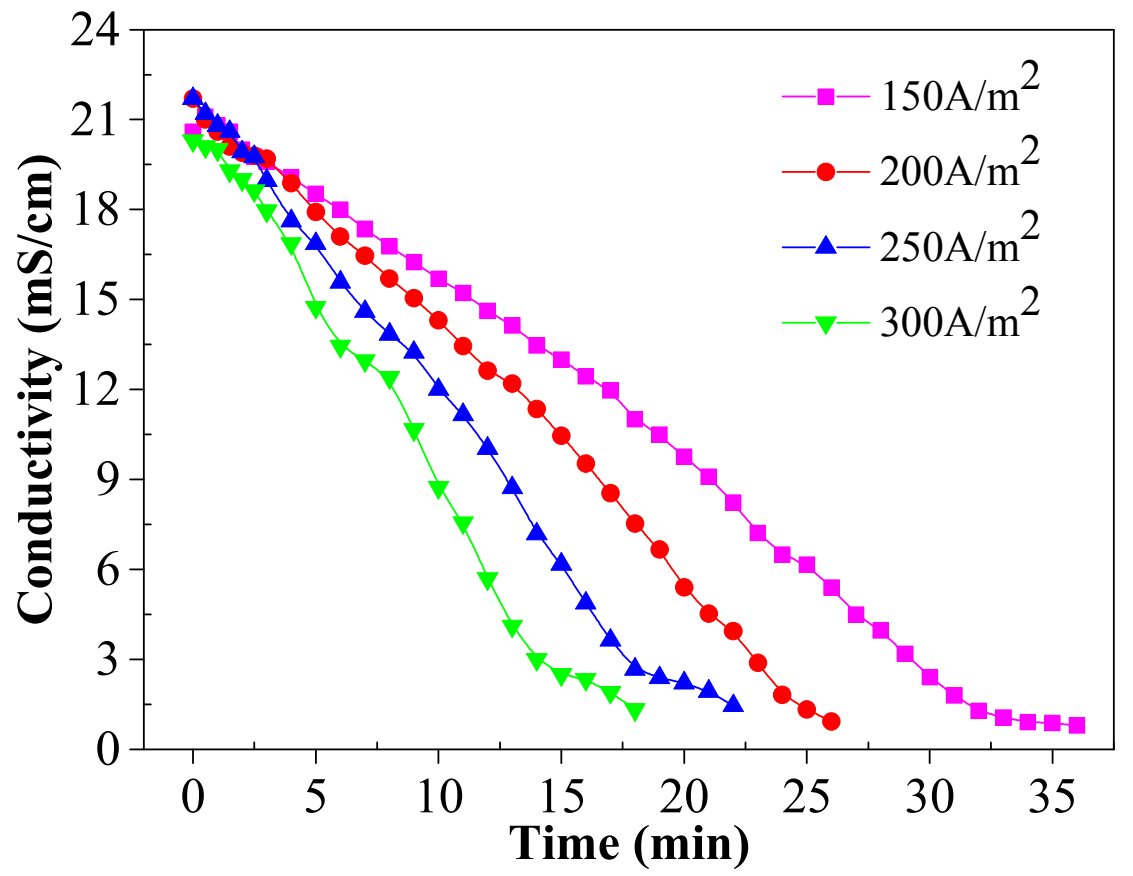

Figure S1 Evolution of conductivity in feed solution during BMED operation at different current densities 